\title{
DECONVOLUTION OF IMAGES FROM BLAST 2005: INSIGHT INTO THE K3-50 AND IC 5146 STAR-FORMING REGIONS
}

\author{
Arabindo Roy ${ }^{1}$, Peter A. R. Ade ${ }^{2}$, James J. Bock ${ }^{3,4}$, Christopher M. Brunt ${ }^{5}$, Edward L. Chapin ${ }^{6}$, Mark J. Devlin ${ }^{7}$, \\ Simon R. Dicker ${ }^{7}$, Kevin France $^{8}$, Andrew G. GibB $^{6}$, Matthew Griffin $^{2}$, Joshua O. Gundersen $^{9}$, Mark Halpern $^{6}$, \\ Peter C. Hargrave ${ }^{2}$, David H. Hughes ${ }^{10}$, Jeff Klein ${ }^{7}$, Gaelen Marsden $^{6}$, Peter G. Martin ${ }^{11}$, Philip Mauskopf ${ }^{2}$, \\ Calvin B. Netterfield ${ }^{1,12}$, Luca Olmi ${ }^{13,14}$, Guillaume Patanchon ${ }^{15}$, Marie Rex ${ }^{7}$, Douglas Scott ${ }^{6}$, \\ Christopher Semisch $^{7}$, Matthew D. P. Truch $^{7}$, Carole TuCKer $^{2}$, Gregory S. Tucker $^{16}$, Marco P. Viero $^{1,4}$, \\ AND Donald V. Wiebe ${ }^{6}$ \\ ${ }^{1}$ Department of Astronomy \& Astrophysics, University of Toronto, 50 St. George Street, Toronto, ON M5S 3H4, Canada; aroy@ cita.utoronto.ca \\ 2 Department of Physics \& Astronomy, Cardiff University, 5 The Parade, Cardiff, CF24 3AA, UK \\ 3 Jet Propulsion Laboratory, Pasadena, CA 91109-8099, USA \\ ${ }^{4}$ Observational Cosmology, MS 59-33, California Institute of Technology, Pasadena, CA 91125, USA \\ ${ }^{5}$ School of Physics, University of Exeter, Stocker Road, Exeter EX4 4QL, UK \\ ${ }^{6}$ Department of Physics \& Astronomy, University of British Columbia, 6224 Agricultural Road, Vancouver, BC V6T 1Z1, Canada \\ ${ }^{7}$ Department of Physics and Astronomy, University of Pennsylvania, 209 South 33rd Street, Philadelphia, PA 19104, USA \\ ${ }^{8}$ Center for Astrophysics and Space Astronomy, University of Colorado, Boulder, CO 80309, USA \\ ${ }^{9}$ Department of Physics, University of Miami, 1320 Campo Sano Drive, Carol Gables, FL 33146, USA
${ }^{10}$ Instituto Nacional de Astrofísica Óptica y Electrónica (INAOE), Aptdo. Postal 51 y 72000, Puebla, Mexico \\ ${ }^{11}$ Canadian Institute for Theoretical Astrophysics, University of Toronto, 60 St. George Street, Toronto, ON M5S 3H8, Canada \\ ${ }^{12}$ Department of Physics, University of Toronto, 60 St. George Street, Toronto, ON M5S 1A7, Canada \\ ${ }^{13}$ Physics Department, University of Puerto Rico, Rio Piedras Campus, Box 23343, UPR station, San Juan, Puerto Rico, USA \\ ${ }^{14}$ Istituto di Radioastronomia, Largo E. Fermi 5, I-50125, Firenze, Italy \\ ${ }^{15}$ Laboratoire APC, 10, rue Alice Domon et Léonie Duquet 75205 Paris, France \\ ${ }^{16}$ Department of Physics, Brown University, 182 Hope Street, Providence, RI 02912, USA \\ Received 2010 October 22; accepted 2011 February 2; published 2011 March 15
}

\begin{abstract}
We present an implementation of the iterative flux-conserving Lucy-Richardson (L-R) deconvolution method of image restoration for maps produced by the Balloon-borne Large Aperture Submillimeter Telescope (BLAST). Compared to the direct Fourier transform method of deconvolution, the L-R operation restores images with bettercontrolled background noise and increases source detectability. Intermediate iterated images are useful for studying extended diffuse structures, while the later iterations truly enhance point sources to near the designed diffraction limit of the telescope. The L-R method of deconvolution is efficient in resolving compact sources in crowded regions while simultaneously conserving their respective flux densities. We have analyzed its performance and convergence extensively through simulations and cross-correlations of the deconvolved images with available high-resolution maps. We present new science results from two BLAST surveys, in the Galactic regions K3-50 and IC 5146, further demonstrating the benefits of performing this deconvolution. We have resolved three clumps within a radius of 4.5 inside the star-forming molecular cloud containing K3-50. Combining the well-resolved dust emission map with available multi-wavelength data, we have constrained the spectral energy distributions (SEDs) of five clumps to obtain masses $(M)$, bolometric luminosities $(L)$, and dust temperatures $(T)$. The $L-M$ diagram has been used as a diagnostic tool to estimate the evolutionary stages of the clumps. There are close relationships between dust continuum emission and both $21 \mathrm{~cm}$ radio continuum and ${ }^{12} \mathrm{CO}$ molecular line emission. The restored extended large-scale structures in the Northern Streamer of IC 5146 have a strong spatial correlation with both SCUBA and high-resolution extinction images. A dust temperature of $12 \mathrm{~K}$ has been obtained for the central filament. We report physical properties of ten compact sources, including six associated protostars, by fitting SEDs to multi-wavelength data. All of these compact sources are still quite cold (typical temperature below $\sim 16 \mathrm{~K}$ ) and are above the critical Bonner-Ebert mass. They have associated low-power young stellar objects. Further evidence for starless clumps has also been found in the IC 5146 region.
\end{abstract}

Key words: stars: formation - submillimeter: general - techniques: image processing

Online-only material: color figures

\section{INTRODUCTION}

The Balloon-borne Large Aperture Submillimeter Telescope flight of 2005 (BLAST05; Pascale et al. 2008; Chapin et al. 2008) conducted both targeted and unbiased surveys in some of the important star-forming regions of the Galactic plane. These were conducted simultaneously at the submillimeter wavelengths of 250,350 , and $500 \mu \mathrm{m}$. BLAST was a precursor to Herschel observations with SPIRE (Griffin et al. 2010). One of the science goals of BLAST surveys was to study the early stages of massive star formation and identify their precursors in order to describe a complete sequence of evolution. In order to realize this ambition, a deep survey complemented by high spatial resolution is required. With a $2 \mathrm{~m}$ telescope, BLAST05 optics were designed to produce dust emission maps at a diffraction-limited resolution of full width at half maximum (FWHM) 40, 58, and $75^{\prime \prime}$ at 250,350 , and $500 \mu \mathrm{m}$, respectively (Pascale et al. 2008). Unfortunately, due to some 
uncharacterized optical problem, the BLAST05 point-spread function (PSF) was anomalous, producing images at a resolution of about 3'.3 (Truch et al. 2008; Roy et al. 2010).

Most of the resulting maps contained clear imprints of the corrupted PSF (hexagonal geometry), indicating the presence of compact sources with angular sizes comparable to the diffraction limit. Measurements of the flux density of a few isolated bright sources can be carried out by aperture photometry or by fitting the PSF. However, because of the large beam area of the corrupted PSF, the sensitivity to compact point sources in the presence of cirrus noise suffers, and the detection of crowded sources is challenging. This leads to an underestimate of the source density and produces an incomplete source list.

In order to improve the image resolution to near the diffraction limit, a direct Fourier transform method of deconvolution was performed in analyzing the Vulpecula region (Chapin et al. 2008). In this method of deconvolution, compact sources in the processed map were enhanced to $1.3,1.6$, and 1.8 at 250 , 350 , and $500 \mu \mathrm{m}$, respectively. However, the deconvolved maps were contaminated by ripples which propagated across the entire map. The generation of ripples is an intrinsic consequence of the direct Fourier inversion method when the PSF varies across the field and is not perfectly known. The amplitude of the ripples is particularly high near bright sources. Strong interference patterns are also observed in the vicinity of multiple bright sources. This makes fainter compact sources hard to detect; furthermore, artificial spatial features are introduced in diffuse structures.

To get the best science return from the BLAST maps, we consider implementation of the Lucy-Richardson (L-R) method of deconvolution (Richardson 1972; Lucy 1974). The L-R deconvolution method is an iterative way of estimating the sky brightness distribution, conserving flux at every successive step. Fortunately, maps from the BLAST 2005 flight have a high signal-to-noise ratio and are well sampled with $15^{\prime \prime}$ pixels, which is particularly important for the performance of L-R deconvolution.

Implementation of L-R deconvolution on the BLAST maps, for example, Cas A (Sibthorpe et al. 2010), Aquila (RiveraIngraham et al. 2010), and Cygnus X (Roy et al. 2011), has remarkably enhanced the compactness of point sources to about $1^{\prime}$ effective resolution. The other benefits of the L-R scheme are: (1) preservation of spatially diffuse structures, (2) suppression of background noise, and (3) resolving blended sources. As an example, in the Cygnus $\mathrm{X}$ region the L-R map led to a source catalog containing 184 sources, significantly greater than the 130 produced using the direct Fourier inversion method.

A basic goal of this paper is to demonstrate the performance of the L-R algorithm in deconvolving BLAST05 maps. We do this via simulations and using "ground truth," i.e., comparing the details in BLAST deconvolved images with available high-resolution maps, thereby establishing the reliability of detected structures, both compact (Section 3.2) and more diffuse (Section 3.3). In those subsections, we focus primarily on the operational consequences of deconvolution for identifying compact sources and defining diffuse structures. We subsequently present new science results made possible by this deconvolution, for the star-forming regions K3-50 (Section 4) and IC 5146 (Section 5). We identify protostellar clumps and characterize their physical properties, such as temperature $(T)$, mass $(M)$, and luminosity $(L)$, by constraining spectral energy distributions (SEDs) using multi-wavelength data. We have also investigated the properties in the "Northern Streamer," a cold molecular ridge in the IC 5146 field.

\section{OVERVIEW OF DECONVOLUTION}

Deconvolution is a common technique used by the astronomical community for enhancing image quality. In the imaging process, various factors are responsible for the distortion of the observed sky map. In the traditional way, the data recorded represent the sky convolved with a PSF. The PSF shape depends not only on the mirror and aperture (window) of the telescope but also often on the atmosphere. Even in an ideal instrumental setup, images are blurred due to secondary peaks of Rayleigh diffraction. In modern astronomy, spatial resolution plays a pivotal role in deciphering morphology and obtaining accurate photometry and hence it is important to try to de-blur images.

Meinel (1986) has shown a wide variety of both linear and nonlinear restoration algorithms, based on maximum likelihood and recursive improvement of images in successive iterations, taking account of the Gaussian and Poisson noise processes. Moreover, the additional a priori information about the positivity of the true image helps to improve resolution. One of the most frequently used methods is the above-mentioned L-R deconvolution scheme, which conserves flux. A key property of this algorithm is that it converges toward the most likely solution of the PSF and image intensity distribution. In the literature, there exist several other techniques for image restoration. A detailed description of the maximum entropy method of deconvolution is given in Narayan \& Nityananda (1986) and Bryan \& Skilling (1980). A maximum correlation method (MCM), which maximizes the correlation between adjacent pixels and simultaneously improves image resolution, has been prescribed by Aumann et al. (1990) for improving IRAS survey maps. This algorithm is also an extension of the L-R solution as described in Meinel (1986) and Velusamy et al. (2008). The latter authors adopted the MCM technique for deconvolution of Spitzer images, improving the resolution by a factor of about three.

These techniques generally require that the PSF be previously determined to high accuracy. When the PSF is only poorly known, an iterative blind deconvolution technique can be used for image restoration, where both the data and the PSF are estimated simultaneously in the successive steps (Fish et al. 1995; Tsumuraya et al. 1994).

\subsection{Two Deconvolution Algorithms}

In the classical approach to the imaging process, the data image $D$ is expressed by

$$
D(x, y)=I(x, y) * P(x, y)+N(x, y),
$$

where $P(x, y)$ is the PSF of the telescope, $I(x, y)$ is the (unknown) unblurred sky image, the symbol $*$ denotes the convolution operator, and $N(x, y)$ is the noise added to the data while imaging. Recursive formulae can be obtained from the maximum-likelihood solution for two different classes of noise distribution, namely the Poisson and the Gaussian noise processes (Meinel 1986; Varosi \& Landsman 1993). From Equations (31) and (64) of Meinel (1986), we write directly the solution for the Poisson noise case in the form (Varosi \& Landsman 1993):

$$
I_{\text {new }}=I_{\text {old }}\left[\left(\frac{D}{I_{\text {old }} * P}\right) * \tilde{P}\right]^{q},
$$


and that for Gaussian noise as:

$$
I_{\text {new }}=I_{\text {old }}+\left(\left[D-\left(I_{\text {old }} * P\right)\right] * \tilde{P}\right)^{q} .
$$

Here, $\tilde{P}$ is the reflected PSF, $\tilde{P}(x)=P(-x)$. For $q=1$, Equation (2) reduces to the L-R algorithm obtained independently from Bayesian statistics (Richardson 1972; Lucy 1974). The Poisson noise process, by its nature, excludes any negative solutions whereas the Gaussian noise process allows negative solutions, subject to the choice of $q$ in Equation (3).

Ideally, after an infinite number of iterations, the output map should converge to the maximum-likelihood solution. However, in practice, after a finite number of iterations in which a close to maximum-likelihood solution has been achieved, the smoothness of the map starts deteriorating (Velusamy et al. 2008). There are no definite and generalized stopping criteria for avoiding this (Prasad 2002). Lucy (1974) recommended a stopping criterion based on a goodness-of-fit test. However, subsequently Lucy (1992) noted that a higher number of iterations might be needed for images with a large number of pixels, and he discouraged the use of the former method.

\subsection{Implementation on BLAST Maps}

BLAST05 raster-scanned targeted regions in the Galactic plane, having different areas ranging from $3 \mathrm{deg}^{2}$ to $10 \mathrm{deg}^{2}$. From the time-ordered data, maps were produced using the optimal map-maker SANEPIC (Patanchon et al. 2008). The combination of high scan speed and low $1 / f$ knee, cross-linking where available, together with common-mode removal in SANEPIC, produces maps retaining diffuse low spatial frequency emission. However, preprocessing of the time-ordered data to remove very low frequency drifts, plus using a low pass cutoff, makes the SANEPIC map average zero-the DC level is not known. The iterative L-R deconvolution algorithm for Poisson noise (Equation (2)) converges only for true positive images. In order to satisfy the positivity of the initial data image $D(x, y)$, we add a constant level to the whole map (a deconvolution operation on a flat map preserves the initial map in its output). The value of the constant is estimated by pixel-pixel correlation of the BLAST images (smoothed to $4^{\prime}$ ) with the corresponding $100 \mu \mathrm{m}$ IRIS image, IRAS data reprocessed by Miville-Deschênes \& Lagache (2005). We carried out deconvolutions with different values of the constant, finding the solution to be robust. After the deconvolution operation, we subtracted the constant from the resulting images.

Special care has been taken to deal with the edges of the scanned region, padding the external area at a value equal to the average around the edge of the map. The convolution operations in the iterative updates were carried out by fast Fourier transform (FFT), and so to maintain a periodic boundary condition and smoothness we apodized the entire map edge with a sine function.

The maps can be produced at various pixelizations, usually $15^{\prime \prime}$, but also $10^{\prime \prime}$ or $20^{\prime \prime}$. This is sufficient to sample the degraded PSF and to sample and recover diffraction-limited information even in the $250 \mu \mathrm{m}$ channel. Our experiments showed that the deconvolution is robust to different pixelizations in this range. Note that the corrupted PSF is not azimuthally symmetric. Therefore, at each passband a synthetic PSF appropriate to the particular scan pattern and coverage of a field is constructed from the well-sampled (10" grid) telescope-frame PSFs of each bolometer (Chapin et al. 2008). In order to avoid artifacts at large angular scales, we have apodized the boundaries of the PSFs with a $\cos ^{2}$ taper from a radius of 4.5-5.7.
For the actual processing we have used the IDL-implemented "Max_likelihood.pro" routine by Varosi \& Landsman (1993), which includes both methods of iterative update, appropriate to Gaussian or Poisson noise. Probably, neither is an accurate noise model for the BLAST Galactic maps, which incorporate a fluctuating cirrus background, bright cirrus structures, and strong compact sources. Empirically, the largest errors in the maps are near the bright sources, this arising from small pointing errors, the asymmetric PSF, and the particulars of the mapmaking process itself. Furthermore, in the reconstruction step implicit in the deconvolution (Equation (1)), whenever the synthetic PSF is not a perfect representation there are larger errors in predicting $D$ where $I$ is brighter. Thus, of the two, the Poisson algorithm is expected to work better because it gives less weight where there are strong point sources, i.e., where there are larger errors in the maps. Although the noise is not actually Poisson and the solution might not be optimal, it is always consistent with the data through Equation (1). Note that a similar situation arises in the HIRES processing of IRAS data, where the noise model is likely a complicated hybrid (Aumann et al. 1990). We have performed experiments with both noise models and, as anticipated, the Poisson alternative was found to perform better in enhancing the structure in the image.

As shown in Figure 1, L-R deconvolution progressively enhances an image, but at the expense of background noise amplification at high frequencies after a large number of iterations. This noise can be suppressed by convolving the deconvolved map with a restoring beam $G$ of FWHM close to the diffraction limit. Mathematically, this step of restoring the image might be achieved in two distinct ways: (1) convolve each side of Equation (1) with $G$, and treat $I * G$ as the unknown sky map to be found by the iterative solution of this new equation; or alternatively, (2) as the effective PSF use $\widehat{(\widehat{P} / \widehat{G}})(x, y)$, where $\widehat{X}$ denotes the Fourier transform of $X$. We have compared the results for these two alternatives with the results of the standard deconvolution subsequently smoothed by the restoring beam and have found that L-R deconvolution gives robust results for all three cases. The images shown in this paper have not been restored.

The reason for this is that despite the complicated and asymmetric PSF and the noise in the maps, application of L-R deconvolution removed the worst effects of the corrupted beam, thus improving significantly the definition of both compact sources and diffuse structures. Compared to the results of the direct inversion, the background obtained is much smoother, although ringing around bright sources has not been completely removed. Such a ringing is not seen in Figure 1, where there are no very bright sources, but can be seen in the maps of Aquila (Rivera-Ingraham et al. 2010), Cygnus X (Roy et al. 2011), and K3-50 (below), each of which contains significantly brighter sources. This same effect is shown in the simulations below.

This ringing is the principal artifact in the deconvolved images, as was also found to be the case in using the HIRES MCM algorithm to produce the Infrared Galaxy Atlas (IGA) from IRAS scans of the Galactic plane (Cao et al. 1997). Velusamy et al. (2008) reduced the ringing artifacts in deconvolutions of Spitzer data by first subtracting the background around the targeted compact sources. However, the large maps from the BLAST surveys are more complex, having large-scale structures of different brightness on which stronger sources are superimposed. Therefore, it is not practical to reduce the background everywhere to be close to 0 . 

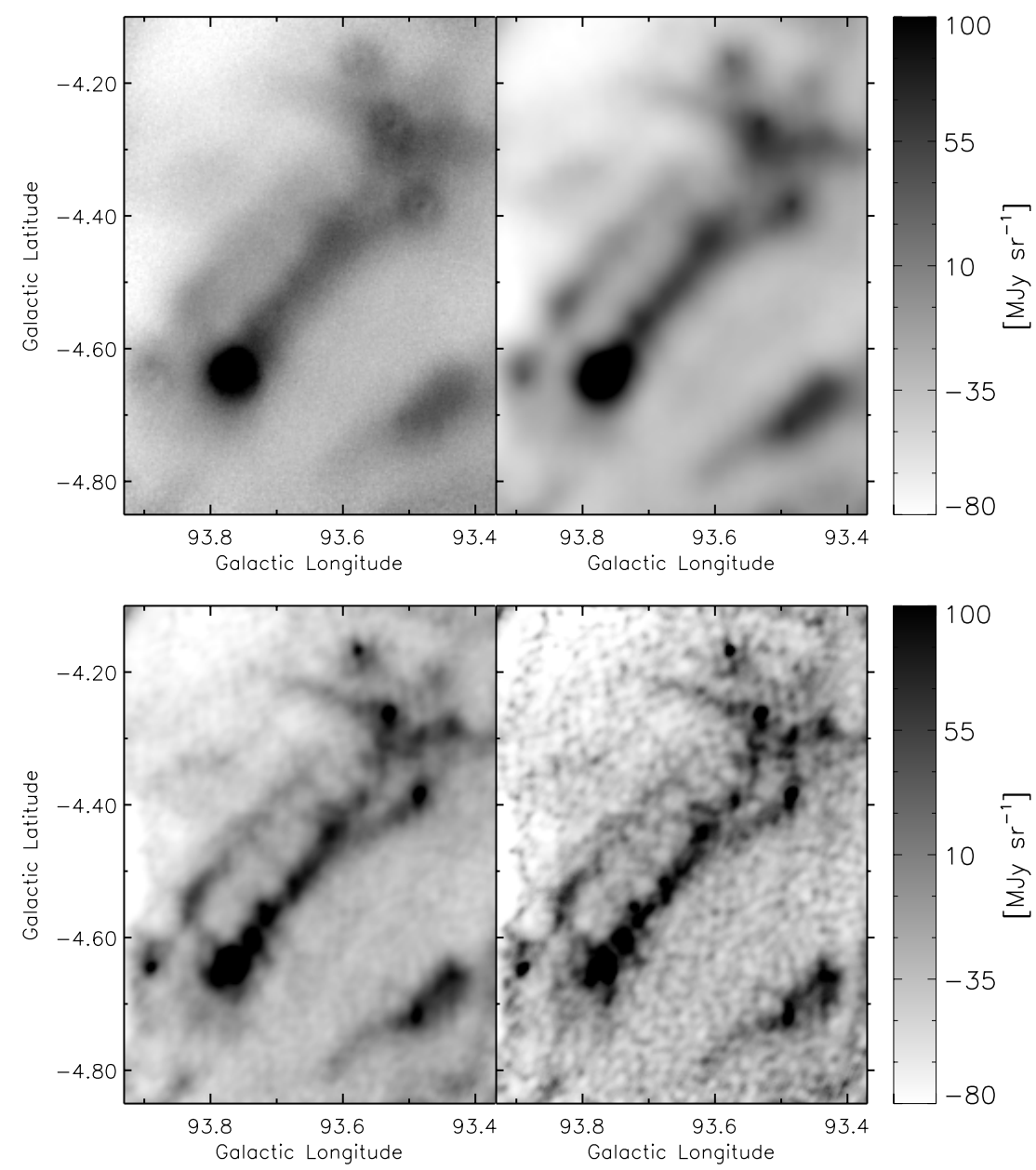

Figure 1. Example of BLAST image deconvolution by the L-R algorithm (Poisson noise), at $250 \mu \mathrm{m}$ for the IC 5146 region. Upper left: unprocessed image where compact sources have the characteristic imprint of the corrupted BLAST PSF. Upper right: deconvolved image after 8 iterations. Lower left and lower right: after 64 and 256 iterations, respectively. The typical size of the compact sources in the final map is about $1^{\prime}$.

These artifacts are on the scale of the corrupted PSF and consist initially of a ring-shaped depression with an outer enhanced ridge. In successive iterative steps the artifact evolves, developing a finer series of depressions and ridges of smaller amplitude spreading from the source out into the rest of the image. The average brightness enclosed within the ringing pattern is very close to the local sky background. The intensity ratio of the brightest, smallest ring to the deconvolved central peak depends somewhat on the local background, but is typically no larger than $0.1 \%$. Nevertheless, near very strong sources it is quite obvious, adding uncertainty in the estimation of the sky background level near bright (often crowded) sources and limiting the detectability of nearby faint sources.

\section{TESTS AND GROUND TRUTH}

\subsection{Simulations}

We have performed simulations to check the robustness of the L-R deconvolution scheme. Fake sources were constructed by convolving normalized narrow Gaussians with the BLAST PSF and then multiplying them with random flux densities. Gaussians with intrinsic FWHMs ranging from 0.5 to $2^{\prime}$ were tested. These simulated sources were inserted at different locations in the map such that the sources were well separated from each other. During the BLAST map-making process, a variance map is obtained from a combination of the noise in the time stream data and the map coverage by the bolometers in the array. We used this variance estimate to add noise to the simulated map, but in these tests we did not take into account the background structures and fluctuations from the diffuse cirrus.

We then deconvolved the simulated map using the PSF and observed the convergence of the measured FWHMs of the sources as a function of iteration, as shown in Figure 2. In these tests we were able to recover the intrinsic size of the sources, convergence occurring after about 64 iterations. In Figure 3, we have plotted the average fractional flux densities recovered at different iterations of the L-R deconvolution. For isolated sources with various FWHMs, about $98 \%$ of the input flux density is recovered. It is possible that the remaining flux density is lost due to the ringing artifact near the source, for which a Gaussian profile used in the fitting is not an accurate model.

Figure 4 shows the performance of L-R deconvolution in a simulation in which two compact sources with relative flux density 10 are placed only 1.5 apart, well within the corrupted PSF. In the blurred map, it is hard to discern a faint second source hidden within the brighter source. However, L-R deconvolution makes it clear. Fitting a double Gaussian to the deconvolved map, we have retrieved the initial flux densities and sizes within $96 \%$ and $99 \%$, respectively. The positions of the recovered sources are accurate within $\sim 1^{\prime \prime}$. 


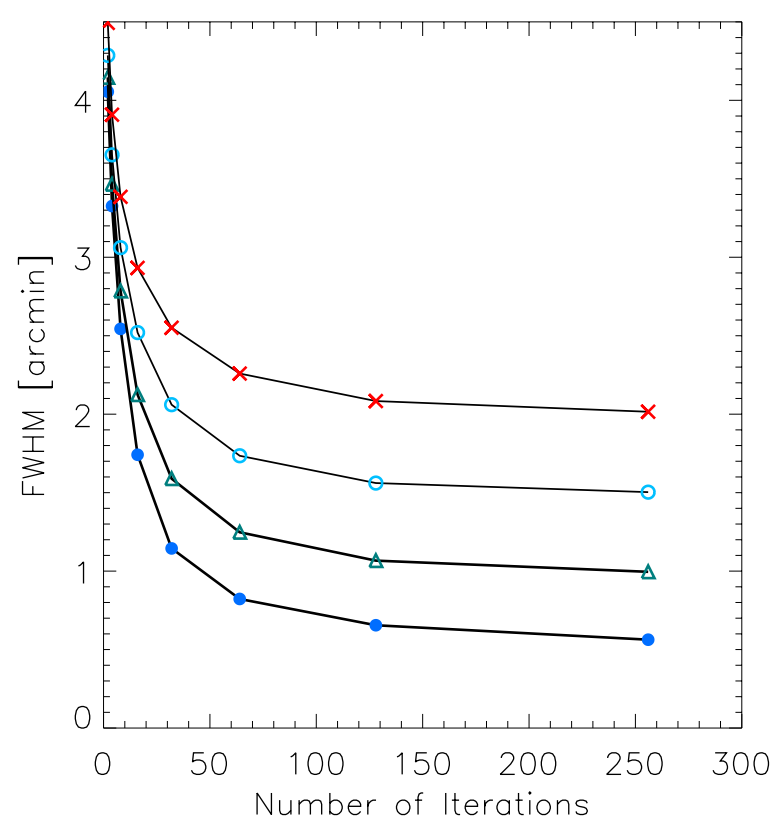

Figure 2. Measured FWHM of simulated sources at successive iterations of L-R deconvolution. Filled circles, triangles, open circles, and crosses represent sources of intrinsic size $0.5,1^{\prime} .0,1^{\prime} .5$, and $2^{\prime} .0$, respectively.

(A color version of this figure is available in the online journal.)

\subsection{L-R Performance on Compact Sources}

Application of the L-R scheme to BLAST05 maps of the Aquila and Cygnus $\mathrm{X}$ regions has already shown impressive improvement in resolving confused sources in crowded regions (Rivera-Ingraham et al. 2010; Roy et al. 2011), thereby enabling the preparation of deeper catalogs. From surveys at both longer and shorter wavelengths, there was abundant ground truth for the fainter sources recovered. To demonstrate the L-R performance on a field of compact sources, we have selected the region near K3-50, which is a young star-forming site containing a group of H II regions. We make use of images from Midcourse Space Experiment (MSX; Mill et al. 1994; Egan et al. 1998) at $8 \mu \mathrm{m}$, the IGA at 60 and $100 \mu \mathrm{m}$, and the CGPS $21 \mathrm{~cm}$ radio continuum survey (Taylor et al. 2003). We also make use of spectral line cubes of ${ }^{12} \mathrm{CO}$ from the Five College Radio Astronomy Observatory (FCRAO) ${ }^{17}$ and of $\mathrm{H}$ I from the CGPS (Taylor et al. 2003). The science results for K3-50 are deferred to Section 4.

Figure 5 shows details of the K3-50 region. The upper left panel shows how the L-R deconvolution operation has improved the resolution (cf. Figure 6 of Truch et al. 2008). For example, the BLAST sources K4 and K5 are now well resolved compact objects in the deconvolved map with an angular resolution of $\sim 40^{\prime \prime}$, which corresponds to a spatial extent of about $1.7 \mathrm{pc}$ at a distance of $8.7 \mathrm{kpc}$. The deconvolution also enabled the detection of faint sources which were otherwise hidden. From "by-eye" examination of the images in all three bands, we identified three sources (K1, K2, and K3) as obvious detections, free of artifacts. Table 1 gives the flux densities and positions and the positions are indicated in all panels of Figure 5. The goal in this section is to find independent confirmation that these are reliable detections with good positional accuracy (we have not attempted to produce a complete source catalog for either of these small fields).

\footnotetext{
17 http://www.astro.umass.edu/ fcrao/
}

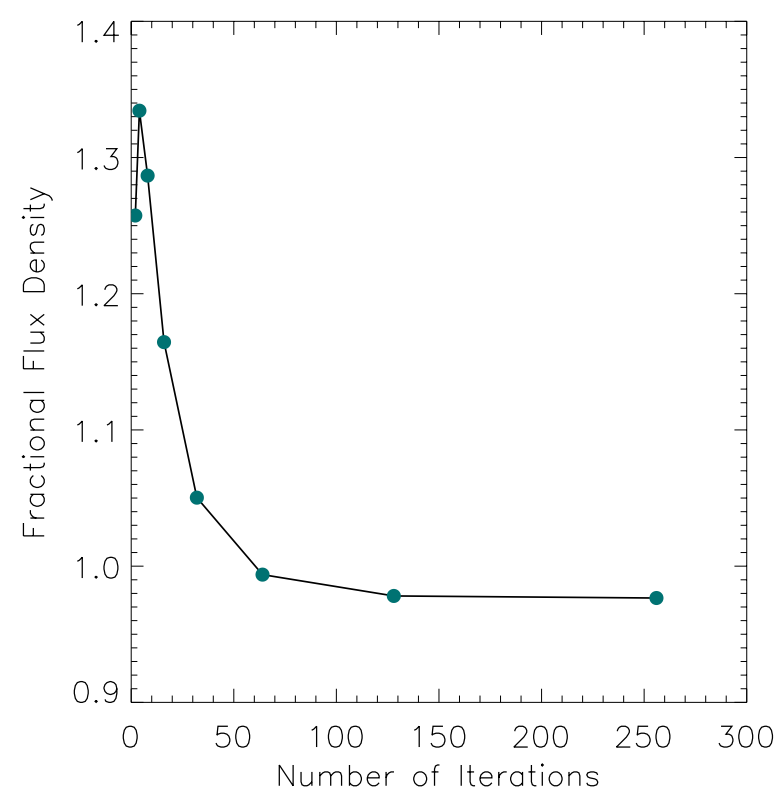

Figure 3. Measured fractional flux density averaged for compact sources with various FWHMs, at successive iterations of L-R deconvolution.

(A color version of this figure is available in the online journal.)

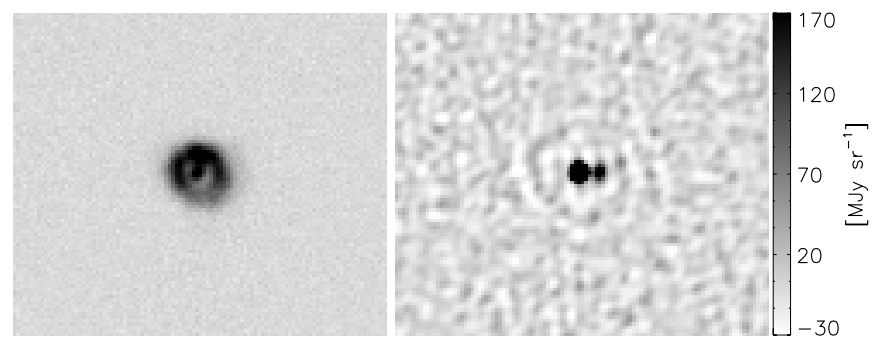

Figure 4. Left panel: two simulated sources of relative flux density 10 placed 1'.5 apart. Right panel: deconvolved map showing the two resolved sources. The brightness scale has been chosen to highlight the faint ring artifact.

The overlaid triangles identify the radio source components obtained by Samal et al. (2010) from $1280 \mathrm{MHz}$ observations, showing that there are multiple sources within the two bright BLAST sources, still unresolved at the improved BLAST resolution. Their names are indicated in the lower left panel, which also shows contours of radio emission. In the lower right panel, we overlay contours of $\mathrm{CO}$ emission integrated over velocity ranging from -30 to $-16 \mathrm{~km} \mathrm{~s}^{-1}$, clearly revealing association of CO clumps with the dust emission. This applies not only to the sources $\mathrm{K} 3, \mathrm{~K} 4$, and $\mathrm{K} 5$, but to more diffuse emission extending out from $\mathrm{K} 4$ and $\mathrm{K} 5$, showing that even this structure near bright sources is not an artifact. The upper right panel shows the same region imaged by $M S X(8 \mu \mathrm{m})$, also at relatively high resolution. Again, there is good correspondence with BLAST.

K3, called IRASB by Samal et al. (2010), coincides with the point source IRAS $19597+3327$ A. At its position there is a CO peak at $-24 \mathrm{~km} \mathrm{~s}^{-1}$, the same velocity as for the much brighter K4 and K5 sources, and in the CGPS H I emission there is an absorption reversal at the same velocity. This indicates that $\mathrm{K} 3$ is related to this complex and is not a galaxy, as it has been alternatively classified (2MASSX J20013735+3335282). The dust emission peak identified as K2 is aligned with an asymmetric ring structure at $8 \mu \mathrm{m}$ with no radio counterpart. $\mathrm{K} 1$ is on an arc of emission seen in the BLAST and MSX $8 \mu \mathrm{m}$ maps, apparently related to the bubble of extended H II 
Table 1

SED Best-fit Parameters of BLAST Sources in the K3-50 Field

\begin{tabular}{|c|c|c|c|c|c|c|c|c|c|c|}
\hline ID & $\begin{array}{c}\text { BLAST Source } \\
\text { Name }\end{array}$ & $\begin{array}{l}S_{250} \\
(\mathrm{Jy})\end{array}$ & $\begin{array}{l}S_{350} \\
(\mathrm{Jy})\end{array}$ & $\begin{array}{l}S_{500} \\
(\mathrm{Jy})\end{array}$ & $\begin{array}{l}S_{100} \\
(\mathrm{Jy})\end{array}$ & $\begin{array}{l}S_{60} \\
(\mathrm{Jy})\end{array}$ & $\begin{array}{c}T \\
(\mathrm{~K})\end{array}$ & $\begin{array}{c}M \\
\left(10^{2} M_{\odot}\right)\end{array}$ & $\begin{array}{c}L \\
\left(10^{4} L_{\odot}\right)\end{array}$ & $\begin{array}{c}L_{\text {bol }} \\
\left(10^{4} L_{\odot}\right)\end{array}$ \\
\hline K1 & $\mathrm{J} 200054+333055$ & $105 \pm 10$ & $61 \pm 5$ & $23 \pm 3$ & $70^{\mathrm{a}}$ & $14^{\mathrm{a}}$ & $20 \pm 1$ & $28 \pm 5$ & $0.7 \pm 0.1$ & $\ldots$ \\
\hline $\mathrm{K} 2$ & $\mathrm{~J} 200112+333802$ & $96 \pm 9$ & $46 \pm 3$ & $13 \pm 1$ & 277 & 175 & $33 \pm 1$ & $6 \pm 1$ & $3 \pm 1$ & $\cdots$ \\
\hline $\mathrm{K} 3^{\mathrm{b}}$ & $\mathrm{J} 200137+333454$ & $144 \pm 20$ & $74 \pm 8$ & $20 \pm 8$ & $\ldots$ & 308 & $34 \pm 1$ & $10 \pm 2$ & $5 \pm 1$ & $\ldots$ \\
\hline K4 & $\mathrm{J} 200146+333214$ & $1468 \pm 19$ & $576 \pm 9$ & $245 \pm 6$ & 11600 & 12000 & $44 \pm 4$ & $68 \pm 14$ & $152 \pm 54$ & 192 \\
\hline K5 & $\mathrm{J} 200154+333343$ & $809 \pm 25$ & $357 \pm 10$ & $184 \pm 6$ & 3300 & 3100 & $35 \pm 2$ & $55 \pm 14$ & $36 \pm 4$ & 39 \\
\hline
\end{tabular}

Notes.

a Data used as upper limit in SED fit.

b IRAS 19597+3327A.
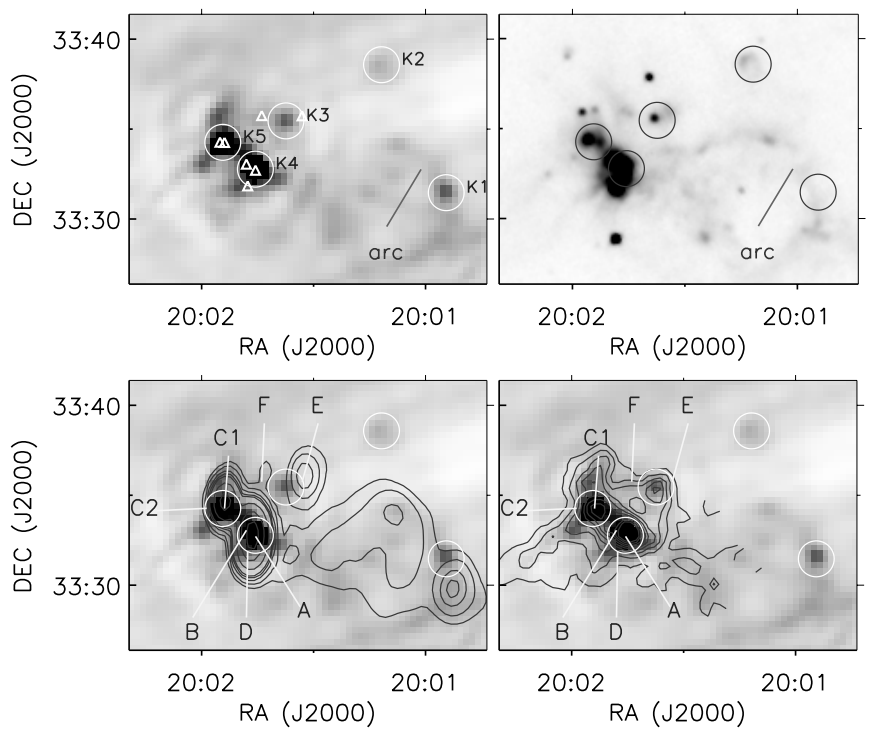

Figure 5. Upper left: BLAST05 map of K3-50 region at $250 \mu \mathrm{m}$ after L-R deconvolution. BLAST compact sources are shown by the circles. The recovered FWHM of K4 (K3-50A) is $36^{\prime \prime}$. Triangles show the positions of radio sources from Samal et al. (2010), with labels A to F (K3-50A to K3-50F) in the lower left panel. Lower left: contours of the $21 \mathrm{~cm}$ radio continuum emission (CGPS) overlaid on the deconvolved $250 \mu \mathrm{m}$ BLAST image. Lower right: contours of the ${ }^{12} \mathrm{CO}$ line emission (FCRAO) integrated over -30 to $-16 \mathrm{~km} \mathrm{~s}^{-1}$. Note that the $\mathrm{CO}$ map coverage does not extend to $\mathrm{K} 1$ and $\mathrm{K} 2$. Upper right: $8 \mu \mathrm{m}$ $M S X$ image. The arc-like feature is also seen in the BLAST image.

emission and its confinement. However, there is no prominent counterpart in either MSX or IRAS images. There is no $21 \mathrm{~cm}$ radio continuum counterpart either, all indicating that the source is cold and unevolved (see below). There is no confirming information from $\mathrm{CO}$, since unfortunately, $\mathrm{K} 1$ (and also K2) is outside the area covered by that emission-line survey; the low-resolution integrated CO map of Dame et al. (2001) hints at an extension in this direction.

Inspection of the deconvolved BLAST images suggests other sources as well. For example, there is a peak in dust emission along the arc, about half way between $\mathrm{K} 1$ and $\mathrm{K} 2$, which coincides with a peak in the radio continuum emission and $M S X$ emission. At $500 \mu \mathrm{m}$ this source is not prominent and so it is probably hot. As mentioned, we have not pursued the study of these fainter sources.

\subsection{L-R Performance on Diffuse Structures}

In this section, we discuss the effectiveness of L-R deconvolution for recovering the morphology of extended structures. BLAST05 mapped a diffuse field toward the Cas A supernova remnant (Sibthorpe et al. 2010). The map was scanned in only

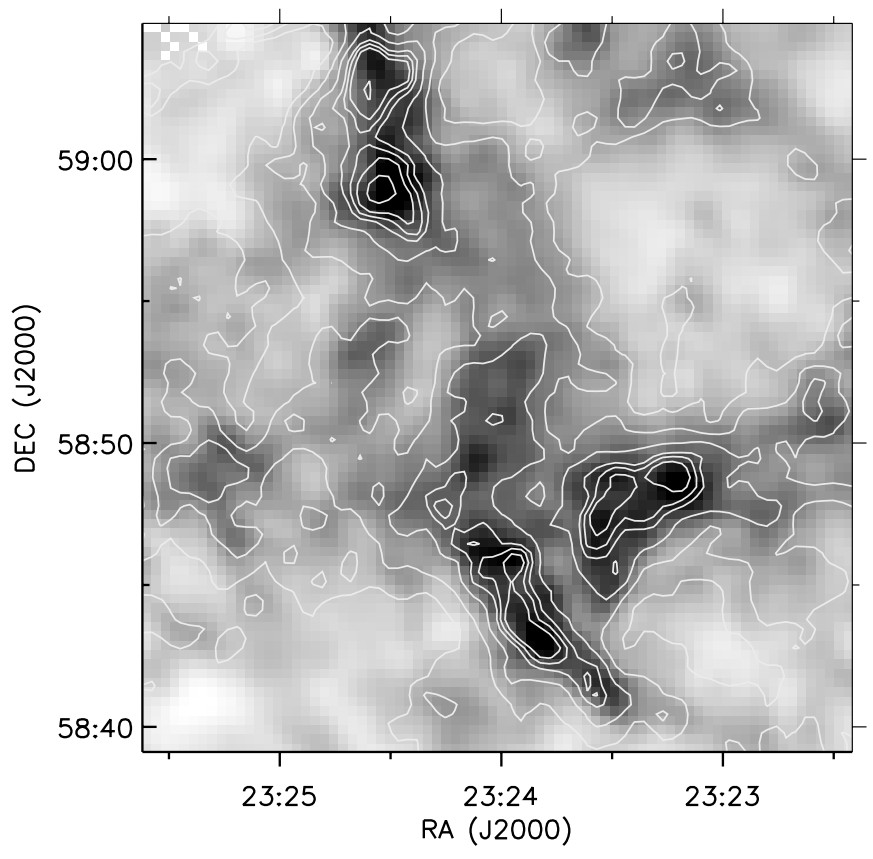

Figure 6. SPIRE $250 \mu \mathrm{m}$ image of the Cas A field convolved with a $40^{\prime \prime}$ Gaussian and reprojected on the 20" BLAST grid. The contour overlay is from the corresponding BLAST deconvolved map, after 32 iterations. After deconvolution, the BLAST image faithfully reveals the morphology of the diffuse structures.

one principal direction, but nevertheless the L-R deconvolution has restored numerous elongated diffuse structures. We have noted how the contrast of these structures increases with successive iterations. However, ultimately the background noise becomes amplified into non-physical small-scale structure, and so intermediate iterations produce the best compromise for studying the details of the diffuse emission. From the appearance of the maps, it was judged that 32 iterations is optimal. Subsequently, this field has been imaged by Herschel ${ }^{18}$ (Barlow et al. 2010), providing the desired ground truth to verify the efficacy of the L-R deconvolution. For comparison, the $250 \mu \mathrm{m}$ SPIRE image was smoothed with a $40^{\prime \prime}$ Gaussian and regridded to the $20^{\prime \prime}$ pixelization of the BLAST image. Figure 6 shows this SPIRE image overlaid with contours from the corresponding deconvolved $250 \mu \mathrm{m}$ BLAST map after 32 iterations. We have again examined the higher-iteration images and confirmed from this direct comparison that for the signal to noise of this BLAST survey, 32 iterations is about optimal.

\footnotetext{
18 http://herschel.esac.esa.int/Science_Archive.shtml
} 


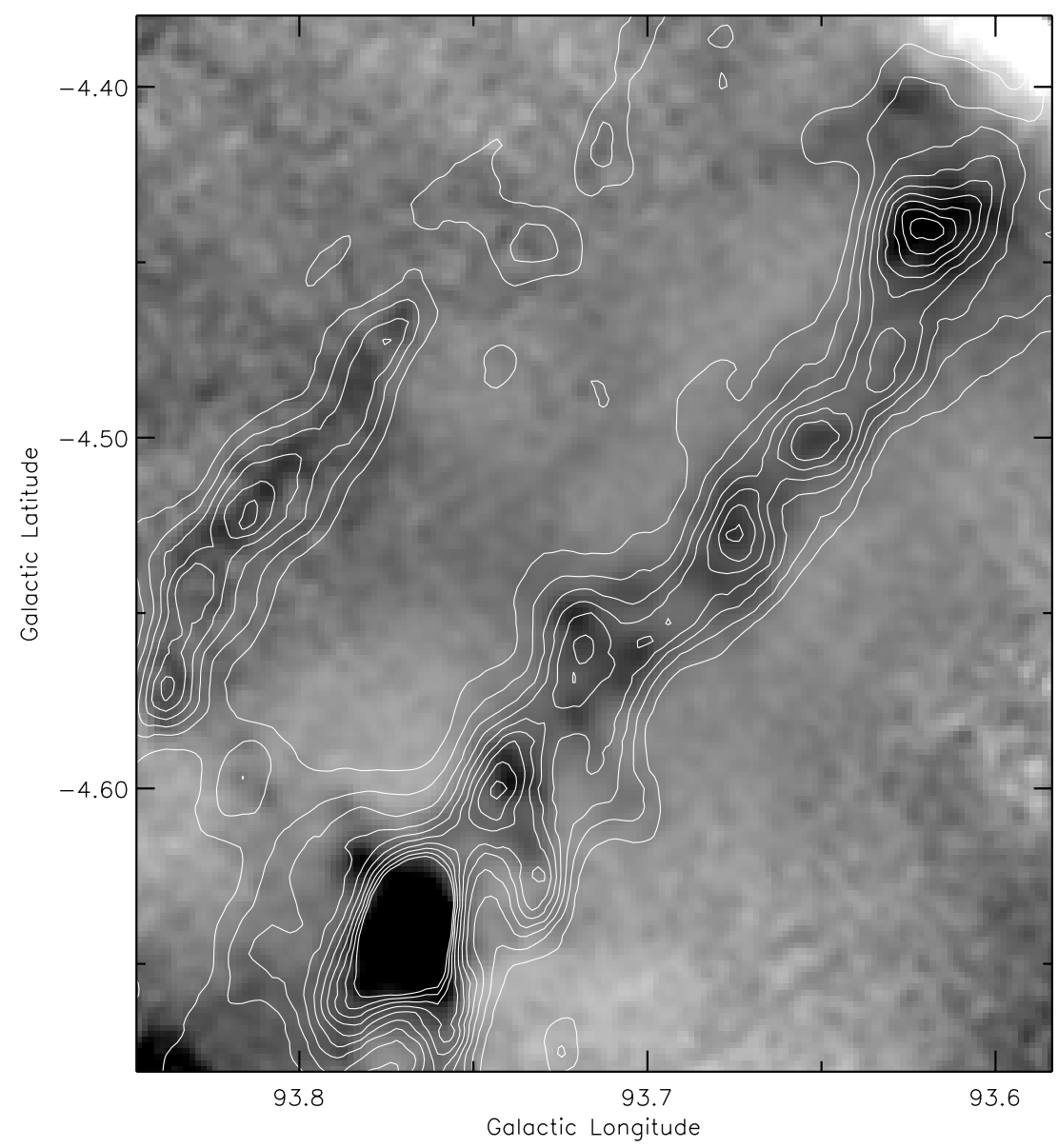

Figure 7. Contours from the deconvolved BLAST $500 \mu \mathrm{m}$ image overlaid on the SCUBA $850 \mu \mathrm{m}$ map of the "Northern Streamer" in the IC 5146 region. BLAST contours trace small-scale sub-structures in the SCUBA map along the filament, again showing the ability of L-R deconvolution to restore morphological detail within diffuse emission.

There is also good evidence for recovering diffuse structures in the fields being studied in this paper. The $M S X 8 \mu \mathrm{m}$ image of the K3-50 region in Figure 5 shows emission (probably from polycyclic aromatic hydrocarbons) along an extended shelllike structure, labeled "arc." Related structure is also seen in the radio emission, suggesting a bubble and surrounding photodissociation region. The deconvolved BLAST05 map reveals that the same structure is also being traced by dust continuum emission, though the effect of the map edge on the lower right is apparent too. Furthermore, there is a faint ridge of submillimeter dust emission extending to the lower left of source K5 (K3-50C) which is traced by the CO contour.

Within the IC 5146 field is an elongated filamentary molecular cloud structure commonly known as the "Northern Streamer." Figure 7 shows a SCUBA $850 \mu \mathrm{m}$ dust map of a targeted section in IC 5146 (Kramer et al. 2003; Di Francesco et al. 2008), overlaid with contours from the $500 \mu \mathrm{m}$ deconvolved image. This shows that the L-R operation has preserved both largescale structures and smaller-scale fragments along the ridge. A discussion of the astrophysics for this region is deferred to Section 5 .

\section{SCIENCE RESULTS FOR K3-50}

$\mathrm{K} 3-50$ is a well-known group of compact $\mathrm{H}$ II regions, namely K3-50A-F, within the star-forming complex W58 (Israel 1976; Howard et al. 1996; Samal et al. 2010). The warm dust surrounding the most prominent ionized region is bright in both far-infrared and submillimeter emission; the correlation with the CGPS $21 \mathrm{~cm}$ radio continuum emission is seen in Figure 5.

The radio sources $\mathrm{K} 3-50 \mathrm{~A}$ and $\mathrm{K} 3-50 \mathrm{~B}$ are well centered inside the BLAST clump K4 (spatial extent $\sim 1.7 \mathrm{pc}$ ), whereas K3-50C is within K5. Samal et al. (2010) further resolved $\mathrm{K} 3-50 \mathrm{C}$ into two sources ( $\mathrm{C} 1$ and $\mathrm{C} 2)$. Moreover, this young star-forming region contains embedded infrared star clusters, namely K3-50D and K3-50B (Bica et al. 2003).

In the overlap region between the BLAST survey and the FCRAO ${ }^{12} \mathrm{CO}$ molecular line survey there is good correspondence between peaks of submillimeter and $\mathrm{CO}$ emission, which provides further insight into global morphology and gas dynamics in the region. The $\mathrm{CO}$ contours in the lower right panel of Figure 5 show that K3, K4, and K5 are part of the same cloud (also see Section 3.2). Velocity peaks associated with these sources are at $-23.6,-24.6$, and $-22.5 \mathrm{~km} \mathrm{~s}^{-1}$, respectively.

In our preliminary work (Truch et al. 2008), we measured flux densities for K3-50A and K3-50C (K4 and K5) by simultaneously fitting a model of two two-dimensional Gaussians convolved with the corrupted BLAST PSF, where sizes, positions, and amplitudes were free parameters of the fit. In our present analysis, with the well-resolved deconvolved map, we fit multiple Gaussians with a linear background model to extract flux densities for individual sources. Table 1 summarizes the flux densities.

In order to achieve a broader multi-wavelength description of the SEDs, we have extracted flux densities at 60 and $100 \mu \mathrm{m}$ from the IGA maps. For longer wavelength data points, 


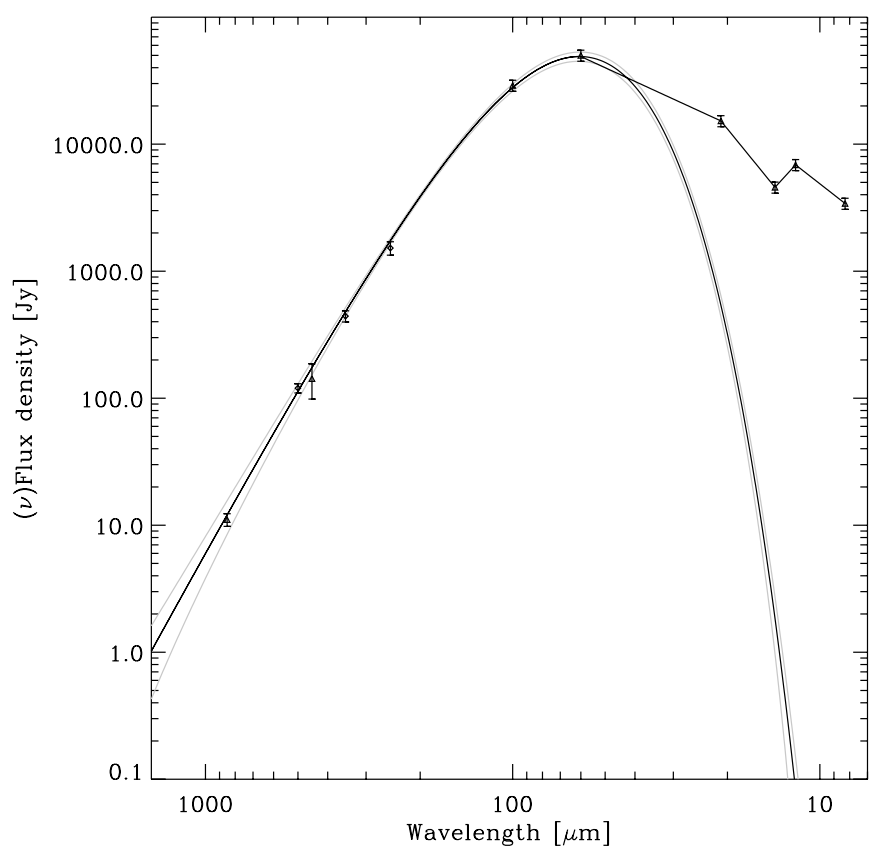

Figure 8. SED for K3-50A (our K4). Flux density multiplied by $v / \nu_{250}$ plotted along the $y$ axis. The solid line is the best fit to the modified blackbody with a fixed dust emissivity parameter of $\beta=1.5$. The gray lines represent an envelope of $68 \%$ confidence level obtained from Monte Carlo simulations. The best-fit parameters are given in Table 1. Data at wavelengths lower than $60 \mu \mathrm{m}$ are not included in the fit but are used in estimating $L_{\mathrm{bol}}$.

we have used the integrated flux densities reported by Thompson et al. (2006) at 450 and $850 \mu \mathrm{m}$ for K4. Due to the restricted coverage of the SCUBA imaging, flux densities at 450 and $850 \mu \mathrm{m}$ are not available for the other sources. We have also used MSX photometric data in deriving the bolometric luminosity; however, we have not used these data in the simple fit to the long-wavelength SED.

The SED-fitting model is a simplified single-temperaturemodified blackbody with a fixed dust emissivity index of $\beta=1.5$. To obtain the mass, we have used Equation (2) of Roy et al. (2011), adopting the same $250 \mu \mathrm{m}$ dust opacity $\kappa_{0}=$ $10 \mathrm{~cm}^{2} \mathrm{~g}^{-1}$ and dust to gas ratio $r=0.01$. We use the commonly adopted distance of $8.7 \mathrm{kpc}$ (Harris 1975; Okamoto et al. 2003; Samal et al. 2010). From the H I absorption velocities and the $\mathrm{CO}$ emission, the sources are in a weak "Perseus arm" feature (in $l-v$ or $b-v$ diagrams) beyond the tangent point (at $\sim 5.5 \mathrm{kpc}$ ), but not beyond the stronger emission at $-60 \mathrm{~km} \mathrm{~s}^{-1}$. Table 1 summarizes the physical properties, namely temperature $(T)$, luminosity $(L)$, and mass $(M)$ for the individual BLAST sources. The reported $1 \sigma$ uncertainties of the physical parameters are obtained from Monte Carlo simulations as described in Chapin et al. (2008).

Figure 8 shows an example of an SED fit, for K4. A best-fit temperature of $44 \pm 4 \mathrm{~K}$ and a total mass of $(7 \pm 1) \times 10^{3} M_{\odot}$ are obtained from the parameters of the fit. Okamoto et al. (2003) have found a star cluster associated with K3-50A (the dominant radio source inside $\mathrm{K} 4$, also an ultracompact $\mathrm{H}$ II region; Kurtz et al. 1994). Our derived luminosity is $(152 \pm 54) \times 10^{4} L_{\odot}$. This clump luminosity is equivalent to a zero-age main sequence (ZAMS) spectral type of $\sim \mathrm{O} 4$ (Panagia 1973), which agrees with Samal et al. (2010), who assigned a spectral type earlier than $\mathrm{O5}$, based on near-infrared measurements. However, the high luminosity supports the possibility of multiple ionizing stars in the stellar cluster. In fact, for K4 it is important to note

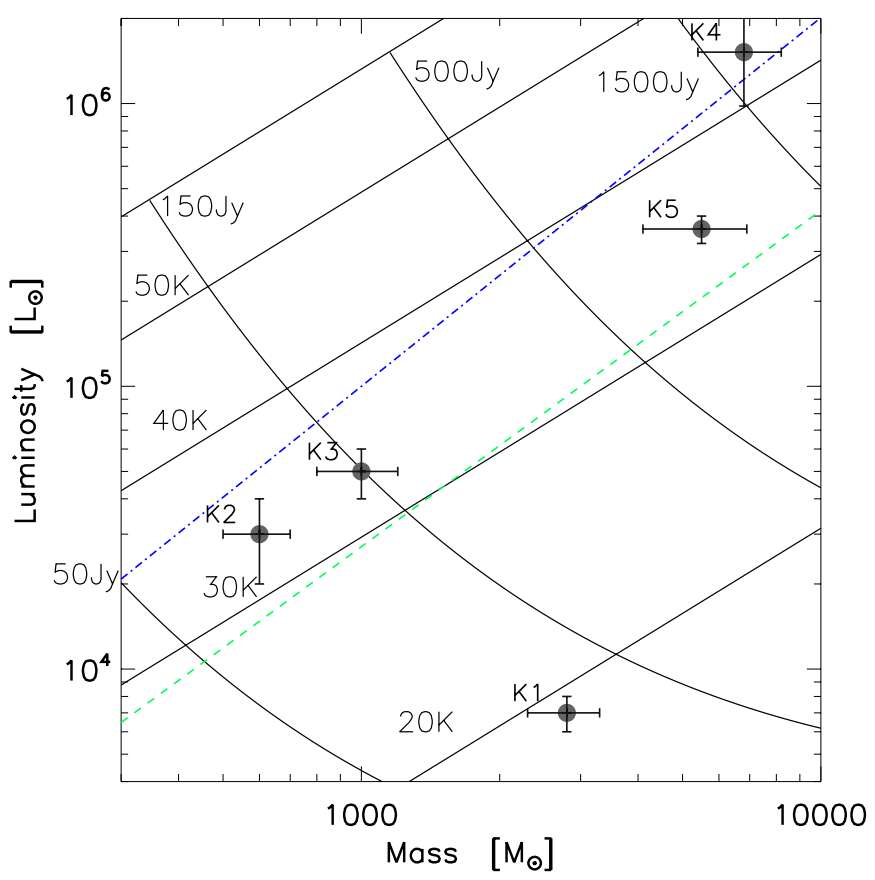

Figure 9. BLAST compact sources in the K3-50 region in the $L-M$ plane. Solid diagonal lines are loci of constant $T$ or $L / M$. Dot-dashed curves orthogonal to these are for constant $250 \mu \mathrm{m}$ flux density. Dash and dot-dashed lines denote sources powered by accretion and nuclear burning, respectively, as derived empirically in Figure 9 of Molinari et al. (2008).

(A color version of this figure is available in the online journal.)

that we measure dust emission whose energy comes collectively from K3-50A and K3-50B.

The position of a source on the $L-M$ plane provides a rough estimate of its evolutionary stage. The relationship of the evolutionary sequence to the underlying energetics powering the dust emission is discussed in detail by Roy et al. (2011). Naively, the evolution in the $L-M$ diagram would be vertical (constant $M)$ as the luminosity increases from accretion onto the massive protostar and the nuclear burning commences. In Figure 9, the dash (green) and dot-dashed (blue) lines are the empirically obtained loci of sources deriving the bulk of their luminosity from accretion and nuclear burning, respectively (Molinari et al. 2008). Figure 9 also confirms that K4 contains one or more ZAMS stars, deriving luminosity mainly from nuclear burning, and hot enough to cause copious ionization.

In a similar fashion, for K3-50C (K5) we obtained a temperature $35 \pm 2 \mathrm{~K}$, which Truch et al. (2008) underestimated due to confusion in the maps combined with the uncertainties in the assumed model. The energetics of $\mathrm{K} 5$ are primarily influenced by the two radio sources inside the clump, namely K3-50C1 and K3-50C2 (Samal et al. 2010). Compared to K3-50A, the dust temperature is somewhat lower, suggesting that $\mathrm{K} 3-50 \mathrm{C}$ is deeply embedded inside the molecular cloud, is less energetic, and is perhaps somewhat less evolved. Integrating the SED for K3-50C, we obtain a luminosity of $(36 \pm 4) \times 10^{4} L_{\odot}$ which is equivalent to $\sim$ O6 on the ZAMS (Panagia 1973). The clump mass is $(55 \pm 14) \times 10^{2} M_{\odot}$, placing it in the appropriate position in the $L-M$ diagram.

$\mathrm{K} 3$ is located between the two radio sources K3-50E and $\mathrm{K} 3-50 \mathrm{~F}$, to its west and east, respectively (see Figure 5). Its luminosity is $(5 \pm 1) \times 10^{4} L_{\odot}$; Samal et al. (2010) underestimated the luminosity $\left(\sim 2.5 \times 10^{3} L_{\odot}\right)$ due to the absence of FIR/submillimeter coverage. The luminosity and 
mass of $(10 \pm 2) \times 10^{2} M_{\odot}$ position $\mathrm{K} 3$ in the $L-M$ plane as a "Class I" object, though with possibly some power coming from accretion. Its equivalent single-star ZAMS spectral type is $\sim$ O8.5, indicating the possibility of ionizing its surroundings. No significant radio continuum emission peak is detected either at $1420 \mathrm{MHz}$ (see Figure 5) or above the $5 \mathrm{mJy}$ level at $1280 \mathrm{MHz}$ (Samal et al. 2010). Possibly there is radio selfabsorption, or the ionizing radiation is absorbed by dust in a dense envelope. Alternatively, the luminosity may come from many slightly lower mass stars, which, being cooler, would produce collectively less ionizing radiation.

A general introduction of $\mathrm{K} 1$ and $\mathrm{K} 2$ was already given in Section 3.2. An accurate distance estimate for these sources is not available. For our present analysis we have assumed a distance of $8.7 \mathrm{kpc}$, similar to the K3-50 region. However, note that the assessment of the evolutionary stages from the $L-M$ diagram is not affected by the distance uncertainty; the position on the $L-M$ plane simply shifts diagonally along a line of constant $L / M$ or temperature. The $\mathrm{K} 2$ clump is associated with emission in the IRAS 60 and $100 \mu \mathrm{m}$ bands and has extended $M S X 8 \mu \mathrm{m}$ emission as well. It is relatively hot, with a temperature of $33 \pm 1 \mathrm{~K}$. The $L-M$ plot suggests that it is a less energetic version of $\mathrm{K} 3$, though the $M S X$ morphology is distinctly different. K1, projected at the outskirts of the K3-50 region, with no young stellar object (YSO) or radio counterpart, is the coldest BLAST clump in this region, at $19 \pm 1 \mathrm{~K}$. In the $L-M$ diagram it is at an earlier stage of evolution, beginning to be powered by accretion.

\section{SCIENCE RESULTS FOR IC 5146}

The IC 5146 molecular cloud complex in Cygnus has been widely studied in the optical, IR (Herbig \& Dahm 2002), submillimeter (Kramer et al. 2003), and molecular lines (Dobashi et al. 1992). Using infrared color excesses in the $J H K$ bands, Lada et al. (1994) produced an extinction map, i.e., the spatial distribution for the dust column density. They also surveyed in the molecular line emission of ${ }^{13} \mathrm{CO}, \mathrm{C}^{18} \mathrm{O}$, and $\mathrm{CS}$. Herbig \& Reipurth (2008) provide a summary of the observational progress achieved in this star-forming region. Harvey et al. (2008) have surveyed the IC 5146 region with Spitzer to study the properties of young stellar objects. Distance estimates range from $460 \mathrm{pc}$ to $1.4 \mathrm{kpc}$ (see Harvey et al. 2008 and references therein). We have adopted $1 \mathrm{kpc}$ from Dobashi et al. (1992), which is close to the 950 pc used by Harvey et al. (2008). Proximity is an advantage for probing the workings of star-forming regions. At this distance, BLAST can resolve spatial structures of about 0.3 pc. Our BLAST05 observation (Figure 10) targeted the filamentary structure of the "Northern Streamer," one of the densest molecular clouds in IC 5146 ("cloud C" of Dobashi et al. 1992), and reportedly the most massive $\left(\sim 2.2 \times 10^{3} M_{\odot}\right)$. The field also contains the dark cloud L1030 and L1031 to the southwest.

\subsection{Compact Sources}

Figure 10 shows the locations of 10 BLAST compact sources in the IC 5146 region. Six of these, with IRAS counterparts, were identified as protostellar candidates by Dobashi et al. (1992). Among these, Elias1-12 (our IC9) is a T Tauri star (Elias 1978), also known to be associated with molecular outflows (Levreault 1983). Follow-up surveys with ${ }^{12}$ CO (Dobashi et al. 1993, 2001) have shown that all of these sources have molecular outflows, revealing the early stages of star formation in progress inside the

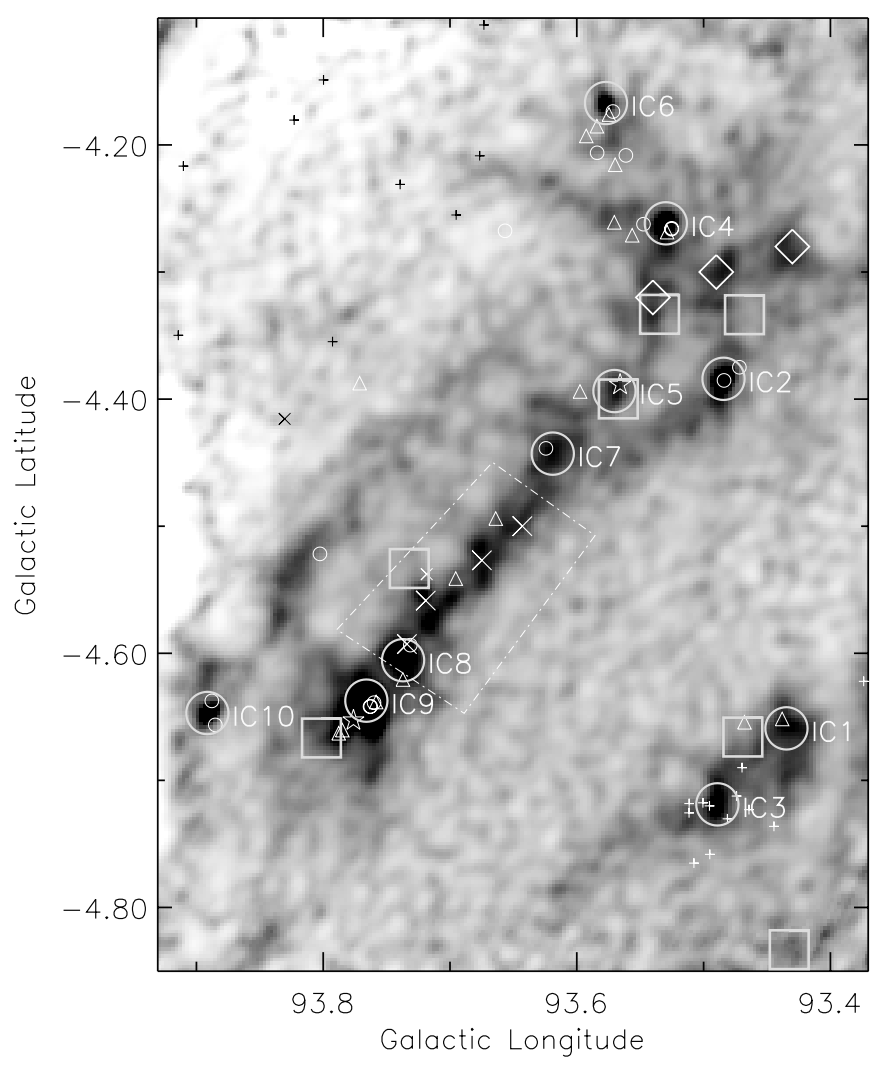

Figure 10. BLAST map of IC 5146 at $250 \mu \mathrm{m}$, showing relationships to other cataloged objects. Circles of radius $1^{\prime}$ locate the BLAST compact sources. Large squares correspond to the peak positions of the velocity-integrated ${ }^{13} \mathrm{CO}$ $(J=1 \rightarrow 0)$ molecular line emission map (Dobashi et al. 1992). YSOs from Harvey et al. (2008) are shown by small circles, stars, triangles, and crosses, to distinguish Class I, flat, II, and III spectra, respectively; the plus symbols represent YSO candidates for which there is no classification. The dust temperature of the cold filament within the outlined dot-dashed box is $11.7 \pm$ $0.4 \mathrm{~K}$ (Section 5.2). Four cores characterized by Kramer et al. (2003) are marked with large crosses. Some additional cold and starless clumps identified by BLAST are marked by large diamonds.

embedding clumps seen by BLAST. The squares in Figure 10 correspond to the peak positions of the velocity-integrated ${ }^{13} \mathrm{CO}$ $(J=1 \rightarrow 0)$ molecular line emission map. There is no direct correspondence between the BLAST dust emission peaks and the ${ }^{13} \mathrm{CO}$ peaks, but the BLAST compact sources are associated with the CO clumps (see Figure 6 of Dobashi et al. 1992).

Harvey et al. (2008) have detected 60 YSO candidates within the region surveyed by BLAST. Using the slopes across MIPS and IRAC data, they classified 38 objects into Class I, flat, II, and III. These relate to progressively later stages of YSO evolution (Evans et al. 2009). Their positions and classifications are given in Figure 10. Along the dense "Northern Streamer," most of the sources belong to Class I and flat classifications, i.e., earlier stages of evolution. Likewise, there are Spitzer YSO counterparts to the 10 BLAST sources, these mostly being Class I. These YSOs within the more extensive BLAST clumps $(0.3 \mathrm{pc})$ are of relatively low power and so the dust is not significantly internally heated and the BLAST dust temperatures are all quite low. For example, IC9 contains several YSOs (in the 2MASS $K$-band image there is reflection nebulosity). At the present submillimeter sensitivity and resolution, not all YSOs have BLAST counterparts.

We have characterized the physical properties of the identified BLAST clumps by fitting SEDs as described in Section 4. Compared to the K3-50 region (dominated by warm dust 
Table 2

Flux Densities of BLAST Sources in the IC 5146 Field

\begin{tabular}{|c|c|c|c|c|c|c|c|c|c|}
\hline ID & $\begin{array}{c}\text { BLAST Source } \\
\text { Name }\end{array}$ & $l$ & $b$ & $\begin{array}{r}F_{250} \\
(\mathrm{Jy}) \\
\end{array}$ & $\begin{array}{l}F_{350} \\
(\mathrm{Jy})\end{array}$ & $\begin{array}{r}F_{500} \\
(\mathrm{Jy})\end{array}$ & $\begin{array}{l}F_{100} \\
(\mathrm{Jy})\end{array}$ & $\begin{array}{l}F_{60} \\
(\mathrm{Jy})\end{array}$ & $\begin{array}{l}F_{24} \\
(\mathrm{Jy}) \\
\end{array}$ \\
\hline IC1 & $\mathrm{J} 214558+471835$ & 93.4334 & -4.6663 & $97.1 \pm 9.0$ & $75.1 \pm 7.3$ & $32.5 \pm 2.1$ & $\cdots$ & $\cdots$ & $\cdots$ \\
\hline $\mathrm{IC} 2^{\mathrm{a}}$ & $\mathrm{J} 214508+473305$ & 93.4842 & -4.3840 & $42.5 \pm 1.5$ & $39.7 \pm 1.4$ & $22.4 \pm 0.8$ & $29.0 \pm 0.5$ & $14.2 \pm 0.1$ & 0.7 \\
\hline IC3 & $\mathrm{J} 214626+471757$ & 93.4844 & -4.7141 & $60.1 \pm 4.7$ & $42.3 \pm 2.3$ & $24.0 \pm 1.0$ & $\ldots$ & $\ldots$ & 0.1 \\
\hline $\mathrm{IC}^{\mathrm{b}}$ & $\mathrm{J} 214452+474026$ & 93.5297 & -4.2616 & $38.4 \pm 2.0$ & $31.5 \pm 1.5$ & $19.9 \pm 1.2$ & $24.3 \pm 0.3$ & $13.3 \pm 0.01$ & 0.8 \\
\hline IC5 & $\mathrm{J} 214534+473559$ & 93.5703 & -4.3936 & $15.0 \pm 1.0$ & $12.1 \pm 0.6$ & $8.7 \pm 0.4$ & $\ldots$ & $\ldots$ & 0.1 \\
\hline $\mathrm{IC}^{\mathrm{c}}$ & $\mathrm{J} 214443+474635$ & 93.5769 & -4.1670 & $45.1 \pm 3.4$ & $33.5 \pm 1.7$ & $19.8 \pm 1.2$ & $10.1 \pm 0.3$ & $2.3 \pm 0.1$ & 0.2 \\
\hline IC7 & $\mathrm{J} 214558+473536$ & 93.6132 & -4.4397 & $69.1 \pm 6.5$ & $47.7 \pm 2.3$ & $30.0 \pm 1.3$ & $20.0 \pm 6.2$ & $1.7 \pm 0.1$ & 0.2 \\
\hline IC8 & $\mathrm{J} 214707+473242$ & 93.7369 & -4.6054 & $23.0 \pm 2.6$ & $20.6 \pm 1.5$ & $11.4 \pm 1.5$ & $\ldots$ & $\ldots$ & $\cdots$ \\
\hline IC $9^{d}$ & $\mathrm{~J} 214722+473221$ & 93.7659 & -4.6372 & $186.6 \pm 3.4$ & $121.8 \pm 2.2$ & $66.9 \pm 1.2$ & $97.5 \pm 10.0$ & $36 . \pm 2.2$ & 2.5 \\
\hline IC10 & $\mathrm{J} 214759+473643$ & 93.8913 & -4.6470 & $45.4 \pm 2.7$ & $30.8 \pm 1.3$ & $14.7 \pm 0.8$ & $12.5 \pm 1.2$ & $2.4 \pm 0.2$ & 0.3 \\
\hline
\end{tabular}

Notes.

a IRAS $21433+4719$.

b IRAS 21429+4726.

c IRAS 21428+4732.

d V1735 Cyg, Elias1-12.

Table 3

SED Best-fit Parameters of BLAST Sources in the IC 5146 Field

\begin{tabular}{lccc}
\hline \hline ID & $T$ & $M_{\text {tot }}$ & $L$ \\
& $(\mathrm{~K})$ & $\left(M_{\odot}\right)$ & $\left(L_{\odot}\right)$ \\
\hline IC1 & $12.7 \pm 1.2$ & $171 \pm 50$ & $48 \pm 16$ \\
IC2 & $16.3 \pm 0.6$ & $35 \pm 8$ & $43 \pm 4$ \\
IC3 & $14.9 \pm 0.1$ & $64 \pm 6$ & $47 \pm 5$ \\
IC4 & $16.1 \pm 0.7$ & $33 \pm 9$ & $38 \pm 4$ \\
IC5 & $14.9 \pm 0.1$ & $15 \pm 2$ & $11 \pm 2$ \\
IC6 & $13.6 \pm 0.3$ & $73 \pm 11$ & $31 \pm 2$ \\
IC7 & $14.0 \pm 0.3$ & $106 \pm 10$ & $53 \pm 3$ \\
IC8 & $15.0 \pm 0.1$ & $25 \pm 3$ & $19 \pm 2$ \\
IC9 & $15.7 \pm 0.3$ & $176 \pm 18$ & $171 \pm 12$ \\
IC10 & $14.3 \pm 0.3$ & $55 \pm 7$ & $31 \pm 2$ \\
\hline
\end{tabular}

emission), the dust temperature of IC 5146 is relatively cold, which motivated us to constrain SEDs with a spectral index of $\beta=2.0$.

Where there are separable IRAS sources, before performing photometry on the BLAST sources, we have convolved the respective maps to the $2^{\prime}$ native beam resolution of the IGA $100 \mu \mathrm{m}$ images (Cao et al. 1997). Otherwise we used the better BLAST resolution. Table 2 summarizes the flux densities. All of these sources have $250 \mu \mathrm{m}$ flux densities greater than at $100 \mu \mathrm{m}$, indicating a relatively cold dust temperature. Constraining the SEDs with the longer wavelength data points is important, but unfortunately SCUBA has observed only a small part of the IC 5146 region (Figure 7). This includes IC7, IC8, and IC9, for which we obtained integrated $850 \mu \mathrm{m}$ flux densities of 10.6, 1.5, and 5.6 Jy, respectively. For the cool sources here we have used only the $100 \mu \mathrm{m}$ flux density in the SED fits, while the 60 and $24 \mu \mathrm{m}$ flux densities provide upper limits, as discussed in Chapin et al. (2008) and Truch et al. (2008). As an example we show the SED for the brightest clump, IC9, in Figure 11.

Table 3 summarizes the physical quantities $T, M$, and $L$ obtained from the best-fit parameters of the SEDs. In this region of IC 5146, the luminosities of the identified BLAST sources range from 11 to $170 L_{\odot}$ and the masses from 15 to $180 M_{\odot}$. The temperatures range from 12.7 to $16.3 \mathrm{~K}$, with an average of $14.8 \mathrm{~K}$.

When a clump is more massive than the Bonner-Ebert critical mass, then gravity overpowers the internal (thermal, magnetic,

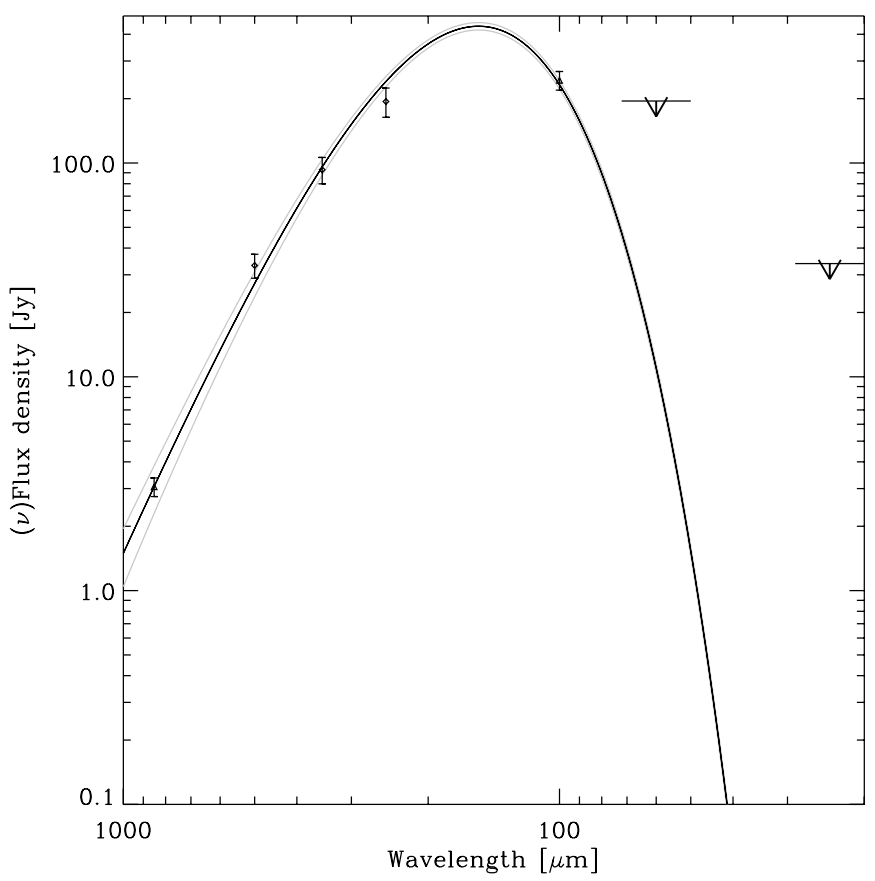

Figure 11. SED as in Figure 8, but for IC9 (Elias1-12) in the IC 5146 region fit with $\beta=2$. Arrows for short-wavelength data represent $3 \sigma$ upper limits above the measured flux density. The best-fit temperature for the cold envelope is $16 \mathrm{~K}$.

and turbulent) supporting pressure and it undergoes collapse. Our BLAST mass estimates appear to be above the critical mass (using the simple prescription described in Kerton et al. 2001), suggesting that the compact sources are gravitationally unstable. Indeed, there are already some YSOs that are generating outflows. Dobashi et al. (2001) showed how physical processes relating to mass, momentum, and energy carried away by the outflows have direct consequences for the stability of the parent cloud and as well influence the evolutionary dynamics of the embedded stars. These outflow parameters have a correspondence with the bolometric luminosity of the accreting source (Dobashi et al. 2001). Certainly feedback processes have already begun in this molecular cloud, and Dobashi et al. (1992) have suggested that outflows have played an important role in supporting the parent cloud from collapsing. 


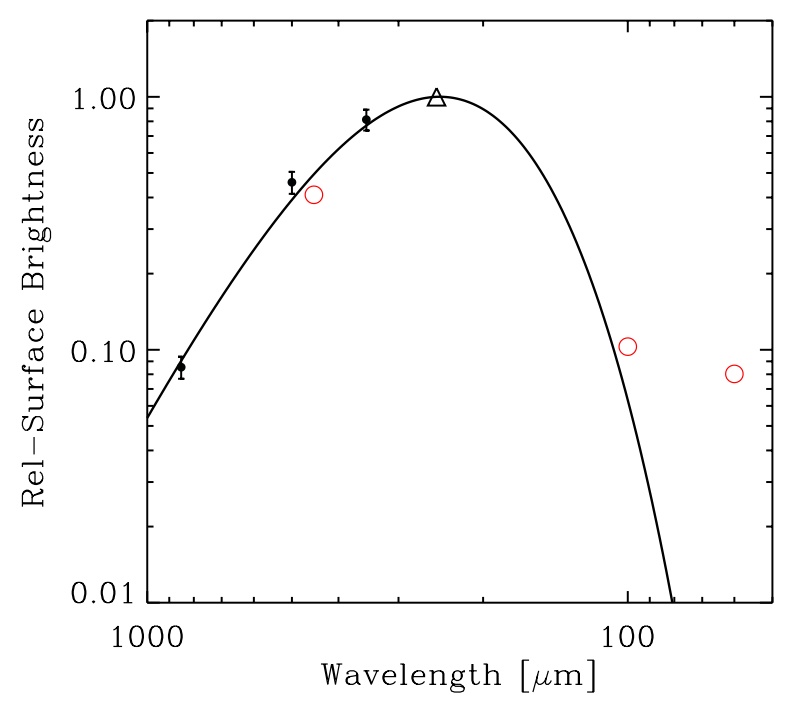

Figure 12. Relative SED obtained from pixel-pixel image correlation with respect to $250 \mu \mathrm{m}$ emission for the area within the dot-dashed box marked in Figure 10. With the dust emissivity index $\beta$ fixed at 2.0, the best-fit temperature is $11.7 \pm 0.4 \mathrm{~K}$. Data points represented by circles are not actually used in the fit, while the triangle shows the value of unity at the normalizing $250 \mu \mathrm{m}$ point. (A color version of this figure is available in the online journal.)

\subsection{Cold Central Filament}

Apart from the above-mentioned compact sources, BLAST observes abundant substructure in the Northern Streamer region. The infrared extinction map by Lada et al. (1994) has also revealed a distribution of high column density clumps throughout this filament. There is a good correlation of $A_{V}$ with the ${ }^{12} \mathrm{CO}$ and ${ }^{13} \mathrm{CO}$ molecular line emission maps by Dobashi et al. (1992). Not unexpectedly, our dust emission map also has a strikingly tight correlation with $A_{V}$ (and $\mathrm{CO}$ which might not be a perfect tracer of column density at large spatial density), and with $850 \mu \mathrm{m}$ emission, as shown in Figure 7.

With earlier SCUBA observations, Kramer et al. (2003) studied a narrow $14^{\prime}$ by 2.5 region extending from IC7 (only at the boundary of their map) to IC8 (included). From the SCUBA colors they found dust temperatures ranging up to $20 \mathrm{~K}$ at the outskirts and between column density peaks and down to $10 \mathrm{~K}$ in "cores" (coincident with peaks in $A_{V}$ ), embedded condensations that effectively shield the interstellar radiation from which the dust derives its power in the absence of a YSO. Note that along the central filament studied there are no embedded YSOs (Figure 10), except at the two ends.

We have estimated a global dust temperature for the portion of the streamer within the box shown in Figure 10, by fitting a relative SED to the pixel-pixel correlation slopes of the image data with respect to $250 \mu \mathrm{m}$. We have used the $850 \mu \mathrm{m}$ SCUBA map to constrain the spectrum at long wavelengths. The filament is not prominent in IRAS bands because the dust is cold. Figure 12 shows the SED. With a dust emissivity index $\beta$ of 2.0 the best-fit temperature is $11.7 \pm 0.4 \mathrm{~K}$, which agrees well with Kramer et al. (2003).

\subsection{Cold Starless Clumps}

Because the BLAST bands are sensitive to colder dust emission, we can detect cold clumps early in their pre-stellar evolution, when their primary energy source is the external radiation field ("stage E" in Roy et al. 2011). Good examples confirmed by BLAST are the four "cores" identified by Kramer et al. (2003) and marked by large crosses in Figure 10. The core numbered 2 by Kramer et al. (2003) is the warmest (18.2 K). This is a complex region near IC8 with large temperature gradients influenced by a YSO visible even to IRAS at short wavelengths. However, the other three cores are cold and apparently starless.

With the larger spatial coverage of the BLAST survey we have identified other cold clumps that have no signs of star formation yet. Some prominent examples are marked by large diamonds in Figure 10, at the positions of the peaks in the $500 \mu \mathrm{m}$ map. Comparing with the visual extinction map of Lada et al. (1994), we find that these BLAST clumps G93.43-4.28, G93.48-4.30, and G93.54-4.33 have $A_{V}$ about 10, 20, and 6 mag, respectively.

The morphology in this field is complex and variable with wavelength because of the different stages of evolution occurring in close proximity. For example, the protostar IC4 occurs at the end of an elongated condensation that has a cold extension in the direction of G93.54 - 4.33. Studies of this region will benefit from the improved resolution of Herschel. With map zero points available from Planck, it will be interesting to map the temperature structure in detail, as has been accomplished in other fields (Bernard et al. 2010; Juvela et al. 2010).

\section{CONCLUSION}

The L-R deconvolution algorithm has been applied successfully to improve BLAST05 images obtained with a corrupted (but known) PSF. This deconvolution has enhanced the raw BLAST map of $\sim 3.3$ resolution to $\sim 1^{\prime}$, near the anticipated diffraction limit. This improves the detectability of faint sources; diffuse structures are revealed in finer detail as well. We have checked via simulations the robustness of applying the L-R scheme, especially the aspects of conserving flux, reliability of restoring intrinsic sizes, and performance in resolving sources in a crowded field. For the actual deconvolved maps of BLAST05 survey fields, we have provided further ground truth for the improved detail in crowded fields and for diffuse structures, by comparing with available multi-wavelength high-resolution images of dust and other tracers.

We have presented science results enabled by the deconvolved maps of two star-forming regions covered by BLAST05, namely K3-50 and IC 5146. We were able to resolve three crowded sources in K3-50, namely K3-50A, K3-50C, and IRASB, and have also characterized another two sources which had previously remained undetected.

The deconvolved maps of IC 5146 have further shown the richness of the field, consisting of both large-scale diffuse structures and compact sources, with a considerable range of dust temperature because of the different stages of evolution. The compact BLAST sources characterized here all have associated YSOs. However, these are not so powerful as to heat up the entire dust clump measured by BLAST, so that $L / M<1 L_{\odot} / M_{\odot}$ and the effective dust temperature is rather low; star formation is just getting under way. In fact, elsewhere there are high column density structures that are starless, like within the central filament in the Northern Streamer, for which we have obtained an average dust temperature of $11.7 \mathrm{~K}$. We have found further examples in the wider BLAST field surveyed.

The BLAST collaboration acknowledges the support of NASA through grant numbers NAG5-12785, NAG5-13301, and NNGO-6GI11G, the Canadian Space Agency (CSA), the UK Particle Physics \& Astronomy Research Council (PPARC), and Canada's Natural Sciences and Engineering Research Council 
(NSERC). We also thank the Columbia Scientific Balloon Facility (CSBF) staff for their outstanding work.

\section{REFERENCES}

Aumann, H. H., Fowler, J. W., \& Melnyk, M. 1990, AJ, 99, 1674

Barlow, M. J., et al. 2010, A\&A, 518, L138

Bernard, J., et al. 2010, A\&A, 518, L88

Bica, E., Bonatto, C., \& Dutra, C. M. 2003, A\&A, 405, 991

Bryan, R. K., \& Skilling, J. 1980, MNRAS, 191, 69

Cao, Y., Terebey, S., Prince, T. A., \& Beichman, C. A. 1997, ApJS, 111, 387

Chapin, E. L., et al. 2008, ApJ, 681, 428

Dame, T. M., Hartmann, D., \& Thaddeus, P. 2001, ApJ, 547, 792

Di Francesco, J., Johnstone, D., Kirk, H., MacKenzie, T., \& Ledwosinska, E. 2008, ApJS, 175, 277

Dobashi, K., Onishi, T., Iwata, T., Nagahama, T., Patel, N., Snell, R. L., \& Fukui, Y. 1993, AJ, 105, 1487

Dobashi, K., Yonekura, Y., \& Hayashi, Y. 2001, PASJ, 53, 811

Dobashi, K., Yonekura, Y., Mizuno, A., \& Fukui, Y. 1992, AJ, 104, 1525

Egan, M. P., Shipman, R. F., Price, S. D., Carey, S. J., Clark, F. O., \& Cohen, M. 1998, ApJ, 494, L199

Elias, J. H. 1978, ApJ, 223, 859

Evans, N. J., et al. 2009, ApJS, 181, 321

Fish, D. A., Brinicombe, A. M., Pike, E. R., \& Walker, J. G. 1995, J. Opt. Soc. Am. A, 12, 58

Griffin, M. J., et al. 2010, A\&A, 518, L3

Harris, S. 1975, MNRAS, 170, 139

Harvey, P. M., et al. 2008, ApJ, 680, 495

Herbig, G. H., \& Dahm, S. E. 2002, AJ, 123, 304

Herbig, G. H., \& Reipurth, B. 2008, in Young Stars and Molecular Clouds in the IC 5146 Region, ed. B. Reipurth (San Francisco, CA: ASP), 108

Howard, E. M., Pipher, J. L., Forrest, W. J., \& de Pree, C. G. 1996, ApJ, 460, 744

Israel, F. P. 1976, A\&A, 48, 193

Juvela, M., et al. 2010, A\&A, 518, L93
Kerton, C. R., Martin, P. G., Johnstone, D., \& Ballantyne, D. R. 2001, ApJ, 552 601

Kramer, C., Richer, J., Mookerjea, B., Alves, J., \& Lada, C. 2003, A\&A, 399, 1073

Kurtz, S., Churchwell, E., \& Wood, D. O. S. 1994, ApJS, 91, 659

Lada, C. J., Lada, E. A., Clemens, D. P., \& Bally, J. 1994, ApJ, 429, 694

Levreault, R. M. 1983, ApJ, 265, 855

Lucy, L. B. 1974, AJ, 79, 745

Lucy, L. B. 1992, A\&A, 261, 706

Meinel, E. S. 1986, J. Opt. Soc. Am. A, 3, 787

Mill, J. D., et al. 1994, J. Spacecr. Rockets, 31, 900

Miville-Deschênes, M.-A., \& Lagache, G. 2005, ApJS, 157, 302

Molinari, S., Pezzuto, S., Cesaroni, R., Brand, J., Faustini, F., \& Testi, L. 2008, A\&A, 481, 345

Narayan, R., \& Nityananda, R. 1986, ARA\&A, 24, 127

Okamoto, Y. K., Kataza, H., Yamashita, T., Miyata, T., Sako, S., Takubo, S., Honda, M., \& Onaka, T. 2003, ApJ, 584, 368

Panagia, N. 1973, AJ, 78, 929

Pascale, E., et al. 2008, ApJ, 681, 400

Patanchon, G., et al. 2008, ApJ, 681, 708

Prasad, S. 2002, J. Opt. Soc. Am., 19, 1286

Richardson, W. H. 1972, J. Opt. Soc. Am. (1917-1983), 62, 55

Rivera-Ingraham, A., et al. 2010, ApJ, 723, 915

Roy, A., et al. 2010, ApJ, 708, 1611

Roy, A., et al. 2011, ApJ, 727, 114

Samal, M. R., et al. 2010, ApJ, 714, 1015

Sibthorpe, B., et al. 2010, ApJ, 719, 1553

Taylor, A. R., et al. 2003, AJ, 125, 3145

Thompson, M. A., Hatchell, J., Walsh, A. J., MacDonald, G. H., \& Millar, T. J. 2006, A\&A, 453, 1003

Truch, M. D. P., et al. 2008, ApJ, 681, 415

Tsumuraya, F., Miura, N., \& Baba, N. 1994, A\&A, 282, 699

Varosi, F., \& Landsman, W. B. 1993, in ASP Conf. Ser. 52, Astronomical Data Analysis Software and Systems II, ed. R. J. Hanisch, R. J. V. Brissenden, \& J. Barnes (San Francisco, CA: ASP), 515

Velusamy, T., Marsh, K. A., Beichman, C. A., Backus, C. R., \& Thompson, T. J. 2008, AJ, 136, 197 